\title{
Usefulness of Presepsin for the Early Detection of Infectious Complications after Elective Colorectal Surgery, Compared with C-Reactive Protein and Procalcitonin
}

\section{Erika Amanai}

Hirosaki University Hospital

Kishiko Nakai ( $\nabla$ kishiko_sk1064@yahoo.co.jp )

https://orcid.org/0000-0002-6618-7803

Junichi Saito

Hirosaki University Hospital

Eiji Hashiba

Hirosaki University Hospital

\section{Takuya Miura}

Hirosaki University Graduate School of Medicine

Hajime Morohashi

Hirosaki University Graduate School of Medicine

Yoshiyuki Sakamoto

Hirosaki University Graduate School of Medicine

Akio Mikami

Hirosaki University Hospital

Kenichi Hakamada

Hirosaki University Graduate School of Medicine

Kazuyoshi Hirota

Hirosaki University Graduate School of Medicine

Research

Keywords: presepsin, infectious complications, colorectal surgery

Posted Date: August 23rd, 2021

DOl: https://doi.org/10.21203/rs.3.rs-812361/v1

License: (c) (i) This work is licensed under a Creative Commons Attribution 4.0 International License. Read Full License 
Version of Record: A version of this preprint was published at Scientific Reports on March 10th, 2022. See the published version at https://doi.org/10.1038/s41598-022-06613-w. 


\section{Abstract}

Background: Infectious complications remain a major clinical problem in colorectal surgery, contributing to prolonged hospital stays, additional costs and significant postoperative mortality. Presepsin has been reported to be a useful marker to diagnose sepsis, similar or superior to procalcitonin (PCT) and Creactive protein (CRP), and plasma presepsin concentrations are associated with the severity of sepsis and its outcome. The aim of this study was to assess the diagnostic value of presepsin in the early detection of infectious complications after elective colorectal surgery, compared with CRP and PCT.

Methods: This study was a prospective observational study. Patients of age $>18$ who underwent elective colon resections were enrolled and patients with end-stage kidney disease were excluded. Blood samples were collected just before surgery and on postoperative day (POD) 1, 2, 3, 4, and 6. Infectious complications were diagnosed by surgeons.

Results: A total of 114 patients were examined, and 27 patients $(23.7 \%)$ developed infectious complications: 11 anastomotic leaks, 13 intra-abdominal infections, and 3 wound infections. CRP and PCT markedly increased from POD1 to POD3 and then gradually decreased toward POD6 in both groups, but the trends of the decrease in the infected group were blunt, compared with those in the non-infected group. On the other hand, presepsin did not show major changes just after surgery, but it increased on POD4 and POD6, when the complications occurred. ROC analysis to predict infectious complications revealed that the best accuracy was obtained on POD 6 for all biomarkers. CRP showed excellent predictability and presepsin showed good predictability. However, the cut-off values of all biomarkers were relatively lower than expected.

Conclusions: The trends of change in presepsin following colorectal surgeries were distinct from those of CRP and PCT. Monitoring the presepsin trends after colorectal surgeries could be helpful to detect postoperative infectious complications.

Trial registration: UMIN000025313. Registered on 17 December 2016.

\section{Background}

Infectious complications of surgery are associated with increased morbidity, length of hospitalization, and health care costs. ${ }^{1-2}$ They are a particular problem in colorectal surgery, a procedure with disproportionately high rates of infectious complication. ${ }^{3-4}$ Early detection and optimal treatment of infectious complications are crucial to improving mortality and morbidity. ${ }^{4-5} \mathrm{C}$-reactive protein (CRP) is probably the most widely used inflammatory biomarker in hospitals worldwide. CRP has been studied as a useful predictor of infectious complications after colorectal resections. Platt et al. reported that CRP measurement on postoperative day (POD) 3 was clinically useful in predicting infectious complications, but its sensitivity and specificity are not very high. ${ }^{6}$ Procalcitonin (PCT), a 116-amino acid peptide, is an inflammatory biomarker with a shorter half-life than CRP. Unfortunately, PCT levels are elevated in 
numerous noninfectious conditions such as trauma, burns and surgical procedures themselves, making accurate interpretation of PCT elevation difficult. ${ }^{7}$

Presepsin is a soluble N-terminal fragment of the cluster of the differentiation marker protein CD14 (SCD14-ST), which is released into the circulation during monocyte activation upon the recognition of lipopolysaccharide from infectious agents. ${ }^{8}$ Several studies have confirmed the usefulness of presepsin as a marker for the diagnosis of sepsis. ${ }^{9-10}$ In studies, plasma presepsin concentrations have been linked the severity of sepsis ${ }^{8,11}$ and its outcome. ${ }^{12}$ Moreover, it has been reported that presepsin levels are not susceptible to severe trauma, burn or invasive surgical procedures, and that, as an early marker of mortality for critically ill patients, it showed better prognostic performance than other biomarkers. ${ }^{13-14}$ However, it remains unknown whether presepsin is useful for the early detection of infectious complications after elective colorectal surgery.

The aim of this study was to assess the diagnostic value of presepsin levels in the early detection of infectious complications after elective colorectal surgery. We compared presepsin in this role with CRP and PCT.

\section{Methods}

\section{Study design}

This study was performed as a prospective, single-center, observational study. The study protocol was approved by our university ethical committee and registered in a publicly accessible database, the UMIN clinical Trial Registry (UMIN000025313).

Patients were enrolled from January 9, to December 31, 2017. Patients 18 years or older who had undergone elective colorectal surgery were enrolled. Patients were excluded if they had obvious signs of infection before surgery or end-stage kidney disease (defined by the Risk-Injury-Failure-Loss-Endstage renal disease classification criteria), as the latter is known to affect presepsin concentrations. ${ }^{15-17}$ Anesthesia was induced and maintained with propofol, remifentanil, ketamine, and rocuronium. The antibiotic prophylaxis consisted of cefotiam hydrochloride $1 \mathrm{~g}$ just before the surgery and every 3 hours after surgery for 24 hours.

\section{Data Collection And Definition Of Complications}

Clinical data were obtained during an initial patient interview and subsequent review of medical documentation. Patient demographic and perioperative data were entered into an electronic databank.

Postoperative infectious complications were classified as surgical site infections (SSIs) and remote infections. SSIs included wound infection, intra-abdominal infection, and anastomotic leakage. Remote infections such as pneumonia, urinary tract infections and central line infections were often exogenous 
and occurred at sites not directly associated with the surgical procedure. ${ }^{18-19}$ Infectious complications were diagnosed by surgeons. Patients underwent postoperative diagnostic tests or treatments only in case of symptoms or signs of infectious complications. Anastomotic leakage was defined as leakage at the site of surgical anastomosis verified either radiologically or upon relaparotomy. Intra-abdominal infection was verified by surgical drainage, imaging study, or by relaparotomy. Wound infection was defined as the presence of pus, either discharged spontaneously or requiring drainage. Pneumonia was diagnosed by the presence of new pulmonary infiltration on chest radiography, accompanied by clinical symptoms and signs. Urinary tract infection was diagnosed by clinical symptoms with a positive urine sediment analysis. Central line infection was diagnosed by clinical symptoms with positive blood cultures and cultures from the catheter tip. ${ }^{20-21}$

\section{Circulating Biomarker Measurement}

Blood samples were collected through an arterial line just before surgery and on POD 1, 2, 3, 4, and 6 to measure plasma levels of presepsin, CRP, and PCT.

Presepsin concentrations were determined by a chemiluminescent enzyme immunoassay. The lower limit of detection was $20 \mathrm{pg} / \mathrm{mL}$. PCT concentrations were determined by an electrochemiluminescent immunoassay, according to the manufacturer's instructions. The lower limit of detection was $0.02 \mathrm{ng} / \mathrm{mL}$. CRP concentrations were measured by a latex coagulation detection method with a nephelometer. The lower limit of detection was $0.02 \mathrm{mg} / \mathrm{dL}$.

\section{Outcomes And Statistical Analysis}

We evaluated the association between circulating biomarkers and infections. More specifically, we assessed the diagnostic value of postoperative presepsin levels in the early detection of infectious complications after elective colorectal surgery, compared with PCT and CRP levels.

In designing the study we calculated the necessary sample size of at least 95 patients to achieve $95 \%$ statistical power at a type $\otimes$ error probability of $0.05 \%$ to detect an AUC of 0.8 , and a rate of SSI or $13 \% .{ }^{2,22-24}$ Anticipating a $20 \%$ loss to follow-up, this number was increased to 114 patients. All statistical calculations were performed using GraphPad Prism version 6.0 (GraphPad Software, La Jolla, CA). Student's t-test was used for the comparison of two groups of parameters that were normally distributed. The Mann-Whitney $U$ test was used to compare differences between two independent groups not normally distributed. Changes of biomarker concentrations over time in both groups were compared using two-way analysis of variance (ANOVA) with repeated measures followed by Bonferroni's multiple comparison test on log-transformed data when appropriate. Predictive performance was assessed by the area under the receiver operating characteristic $(\mathrm{ROC})$ curves. A p value $<0.05$ was accepted as significant. 


\section{Results}

\section{The demographic data of the patients (Table 1)}

A total of 114 patients were included in this study, and 27 patients (23.7\%) developed infectious complications. The clinical characteristics of the 114 patients are shown in Table 1. The complications included 11 anastomotic leakages, 13 intra-abdominal infections and 3 wound infections. Among those 27 patient with complications, one coincidentally had a urinary tract infection. The median interval between surgery and the diagnosis of infection was 5 days (Min-Max days: 3-9 days). Six patients required additional operations: 4 ileo-colostomies, 1 Hartmann resection and 1 re-suturing of the anal region.

The patients who underwent rectal resections had significantly more complications than the patients who did not undergo rectal surgery $(74.0 \%$ vs $49.4 \%, p=0.024)$. The length of hospital stay was significantly longer in patients with complications than in patients without complications $(24.5 \pm 29.6$ vs. $12.6 \pm 7.1, p$ $<0.001$ ), implying higher costs of medical treatment for patients with complications. There was no readmission due to morbidities within the first 30 days after surgery.

\section{Time course of presepsin, CRP and procalcitonin levels during the study period}

Changes in the three biomarkers are shown in Figure. There were significant differences in presepsin, CRP and PCT concentrations between the infected and non-infected groups (each P value for time-infected interaction was less than 0.0001). CRP and PCT significantly and markedly increased from POD1 to POD3 and then gradually decreased toward POD6, but the decreases in the infected group were more gradual than those in the non-infected group. On the other hand, presepsin did not increase in either group within the first 3 days after the surgery, but the infected group showed a significant increase on POD4 and POD6, compared with the non-infected group. The shapes of the trend of presepsin were clearly different from those of the other two markers, CRP and PCT.

\section{Prognostic Accuracy Of Presepsin, Crp And Pct}

The results of ROC analysis defining the area under the curve (AUC) of the three biomarkers for infectious complications are shown in Table 2. ROC analysis to predict infectious complications revealed that the best accuracy was obtained on POD 6 for all biomarkers. The cut-off values, sensitivities, specificities and AUCs are also shown in Table 2. The AUC values of CRP, presepsin and PCT on POD 6 were 0.91, 0.82 and 0.75 , respectively.

\section{Discussion}

This study showed the unique behaviors of presepsin in colorectal surgery patients with and without infectious complications, compared with CRP and PCT. CRP and PCT significantly increased immediately 
after the surgery and gradually decreased several days after the surgery. While the elevations of CRP and PCT were relatively prolonged in infected groups, compared with non-infected groups.

CRP is an inflammatory biomarker that increases in response not only to infection but also to inflammation caused by non-infectious stimuli. It is widely used as a predictor of infectious complications after surgery. Platt et al. reported that CRP measurement on POD3 is clinically useful for predicting infectious complications. ${ }^{6}$ PCT has been also reported to be useful as a septic biomarker ${ }^{25}$, but PCT levels are also known to be elevated in non-infectious conditions such as trauma, burn and even surgical procedures. ${ }^{7,}{ }^{26}$ In this study the levels of median PCT concentrations in both infected and noninfected groups were higher than $1 \mathrm{ng} / \mathrm{ml}$, which was more than the reported cutoff values of $0.5 \mathrm{ng} / \mathrm{ml}$ for suspecting bacterial infections. ${ }^{25,27}$ Overall, PCT may not be very useful for detecting infectious complications in colorectal surgery because PCT is so easily increased, the levels of increase vary from patient to patient, and the AUC of PCT on POD 6 was 0.75 , indicating less than desired predictability. In contrast to CRP and PCT, presepsin markedly increased only in response to the infectious insults after surgery, with good predictability shown by an AUC of 0.82 on POD 6. Presepsin has been shown to be released into the blood circulation during monocyte activation upon the recognition of lipopolysaccharides from infectious agents. ${ }^{8}$ Several studies have confirmed the usefulness of presepsin as a biomarker for the diagnosis of sepsis. ${ }^{9-10}$ Our study showed the usefulness of presepsin to detect infectious complications in colorectal surgery in line with those previously reported studies.

The cutoff value of presepsin to detect infection on POD 6 was $299 \mathrm{pg} / \mathrm{ml}$, less than previously reported values. Popv and colleague ${ }^{28}$ reported that the cutoff value of presepsin to identify infection after cardiac surgery was $702 \mathrm{pg} / \mathrm{ml}$, with a sensitivity of $72 \%$ and a specificity of $66 \%$. Endo and colleagues ${ }^{10}$ reported that the cutoff value of presepsin to discriminate bacterial from nonbacterial infectious disease was $600 \mathrm{ng} / \mathrm{l}$, with a sensitivity of $87.8 \%$ and specificity of $81.4 \%$. On the other hand, Liu and colleagues ${ }^{14}$ reported that the cutoff value of presepsin for diagnosing sepsis was $317 \mathrm{pg} / \mathrm{ml}$, with a sensitivity of $70.8 \%$ and specificity of $85.8 \%$, and that the median [25th to 75 th percentiles] presepsin concentration in healthy individuals was $130[104,179] \mathrm{pg} / \mathrm{ml}$. Tsuchida and colleagues ${ }^{29}$ reported the cutoff values of presepsin for detecting bacterial infection and bacteremia in non-severe outpatients were $226 \mathrm{pg} / \mathrm{ml}$ (sensitivity $67 \%$, specificity $66 \%$ ) and $256 \mathrm{pg} / \mathrm{ml}$ (sensitivity $84 \%$, specificity $70 \%$ ). The latter two studies, as well as our own, suggest that the optimal cutoff value of presepsin to detect an infectious complication may not be as large as expected.

In our study, CRP had the highest sensitivity of $81.5 \%$ and specificity of $88.4 \%$ on POD6, with an AUC of 0.91 , which was higher than that of presepsin, suggesting that CRP might be better at detecting postoperative infections in colorectal surgery. However, the cutoff value of CRP was $7.39 \mathrm{mg} / \mathrm{dl}$, which was not extraordinary high and much lower than the peak CRP level after surgery even in the non-infected group. In order to use the cutoff value of CRP in POD6, it's important to know its entire post-surgical trajectory. 
Our study has several limitations, including its single-center design. First, we did not examine the correlation between postoperative renal function and biomarker concentrations. We simply excluded patients with diagnosed end-stage kidney disease, knowing that renal function can affect presepsin concentrations. Even though there were no patients in this study who required renal replacement therapy during our observation, it was known that presepsin and PCT concentrations are significantly correlated with the serum creatinine concentration after cardiac surgery. ${ }^{30}$ Thus, the postoperative course of renal function might have affected biomarker concentrations in this study. Second, we need to consider the effects of reoperation. Six patients required additional operations for postoperative infectious complications in this study. Second invasive surgical procedures might have affected changes in biomarker concentrations during the observation period.

\section{Conclusion}

The trend of change in presepsin levels following colorectal surgery was distinct from those of CRP and PCT. Our data suggest that monitoring presepsin concentrations after colorectal surgery is helpful for detecting early postoperative infectious complications.

\section{Declarations}

\section{Ethics approval and consent to participate}

This study protocol was approved by our university ethical committee and registered in a publicly assessable database, the UMIN clinical Trial Registry (in the Japan Primary Registries Network; UMIN000025313).

\section{Consent for publication}

Not applicable.

\section{Availability of data and materials}

All data generated or analysed during this study are included in this published article.

\section{Competing interests}

LSI Medience Corporation provided the kits for measuring presepsin (PATHFAST; Mitsubishi Chemical Medience Corporation, Tokyo) free of cost.

\section{Funding}

This study was supported by the Grant-in-Aid for Young Scientists (B) of the Japan Society for the Promotion of Science (JP19K18259).

\section{Authors' contributions}


EA designed the study, collected and evaluated the data. $\mathrm{KN}$ designed the study, analyzed and interpreted the data, and drafted the manuscript. JS designed the study, analyzed and interpreted the data. EH designed the study, reviewed the analysis of the data and edited the manuscript. TM, HM, YS and KH helped conduct the study. AM measured and collected the data. $\mathrm{KH}$ made substantial contributions to the conception and design of the study and helped with manuscript writing and statistical analysis. All authors have read and approved the final manuscript.

\section{Acknowledgement}

This study was partially supported by grants from the 2017-2018 Hirosaki University Work-Life Balance Support Program for Researchers and JSPS KAKENHI Grant Number JP19K18259.

\section{Authors' information}

${ }^{1}$ Department of Anesthesiology, Hirosaki University Hospital,

${ }^{2}$ Division of Intensive Care Unit, Hirosaki University Hospital, Hirosaki, Japan

${ }^{3}$ Department of Gastroenterological Surgery and Pediatric Surgery, Hirosaki University Graduate School of Medicine, Hirosaki, Japan

${ }^{4}$ Central Clinical Laboratory, Hirosaki University Hospital, Hirosaki, Japan

${ }^{5}$ Department of Anesthesiology, Hirosaki University Graduate School of Medicine, Hirosaki, Japan

\section{References}

1. Wick EC, Hirose K, Shore AD, Clark JM, Gearhart SL, Efron J, et al. Surgical site infections and cost in obese patients undergoing colorectal surgery. Arch Surg. 2011; 146:1068-72.

2. de Lissovoy G, Fraeman K, Hutchins V, Murphy D, Song D, Vaughn BB. Surgical site infection: incidence and impact on hospital utilization and treatment costs. Am J Infect Control 2009; 37: 38797.

3. Wick EC, Vogel JD, Church JM, Remzi F, Fazio VW. Surgical site infections in a "high outlier" institution: are colorectal surgeons to blame? Dis Colon Rectum. 2009; 52:374-9.

4. Bratzler DW, Hunt DR. The Surgical Infection Prevention and Surgical Care Improvement Projects: national initiatives to improve outcomes for patients having surgery. Clin Infect Dis. 2006; 43:32230 .

5. Keenan JE, Speicher PJ, Thacker JK, Walter M, Kuchibhatla M, Mantyh CR. The preventive surgical site infection bundle in colorectal surgery: an effective approach to surgical site infection reduction and health care cost savings. JAMA Surg. 2014; 149: 1045-52. 
6. Platt JJ, Ramanathan ML, Crosbie RA, Anderson JH, McKee RF, Horgan PG, et al. C-reactive protein as a predictor of postoperative infective complications after curative resection in patients with colorectal cancer. Ann Surg Oncol 2012; 19: 4168-77.

7. Becker KL, Snider R, Nylen ES. Procalcitonin assay in systemic inflammation, infection, and sepsis: Clinical utility and limitations. Crit Care Med. 2008; 36: 941-52.

8. Shozushima T, Takahashi G, Matsumoto N, Kojika M, Endo S, Okamura Y. Usefulness of presepsin (sCD14-ST) measurements as a marker for the diagnosis and severity of sepsis that satisfied diagnostic criteria of systemic inflammatory response syndrome. J Infect Chemo 2011; 17: 764-9.

9. Yaegashi Y, Shirakawa K, Sato N, Suzuki Y, Kojika M, Imai S, et al. Evaluation of a newly identified soluble CD14 subtype as a marker for sepsis. J Infect Chemo 2005; 11: 234-8.

10. Endo S, Suzuki Y, Takahashi G, Shozushima T, Ishikura H, Murai A, et al. Usefulness of presepsin in the diagnosis of sepsis in a multicenter prospective study. J Infect Chemo 2012; 18: 891-7.

11. Kojika M, Takahashi G, Matsumoto N, Kikkawa T, Hoshikawa K, Shioya N, et al. Serum levels of soluble CD14 subtype reflect the APACHE $\triangle$ and SOFA scores. Medical Postgraduatcs 2010; 48: 4650 .

12. Endo S, Suzuki Y, Takahashi G, Shozushima T, Ishikura H, Murai A, et al. Presepsin as a powerful monitoring tool for the prognosis and treatment of sepsis: a multicenter prospective study. J Infect Chemo 2014; 20: 30-4.

13. Masson S, Caironi P, Spanuth E, Thomae R, Panigada M, Sangiorgiet G, et al. Presepsin (soluble CD14 subtype) and procalcitonin levels for mortality prediction in sepsis: data from the Albumin Italian Outcome Sepsis trial. Crit Care 2014; 18: R6.

14. Liu B, Chen YX, Yin Q, Zhao YZ, Li CS. Diagnostic value and prognostic evaluation of presepsin for sepsis in an emergency department. Crit Care 2013; 17: R244.

15. Saito J, Hashiba E, Kushikata T, Mikami A, Hirota K. Changes in presepsin concentrations in surgical patients with end-stage kidney disease undergoing living kidney transplantation: a pilot study. J Anesth 2016; 30: 174-7.

16. Nagata T, YasudaY, Ando M, Abe T, Katsuno T, Kato S, et al. Clinical impact of kidney function on presepsin levels. PloS One 2015; 10: e0129159.

17. Nakamura Y, Hoshino K, Kiyomi F, Kawano Y, Mizunuma M, Tanaka J, et al. Comparison of accuracy of presepsin and procalcitonin concentrations in diagnosing sepsis in patients with and without acute kidney injury. Clin Chim Acta; 490. 200-6.

18. Horan TC, Gaynes RP, Martone WJ, Jarvis WR, Emori TG. CDC definitions of nosocomial surgical site infections, 1992; A modification of CDC definitions of surgical wound infections. Infection Control Hosp Epidemiol 1992; 13: 606-8.

19. Mangram AJ, Horan TC, Pearson ML, Silver LC, Jarvis WR. Guideline for Prevention of Surgical Site Infection, 1999. Centers for Disease Control and Prevention (CDC) Hospital Infection Control Practices Advisory Committee. Am J Infect Control 1999; 27: 97-132. 
20. Watanabe M, Kusachi S. SURVEILLANCE OF PERIOPERATIVE INFECTIONS AND INFECTION CONTROL. Nihon Geka Gakkai Zasshi. 2016; 117: 199-203. Japanese.

21. Grant MC, Yang D, Wu CL, Makary MA, Wick EC. Impact of Enhanced Recovery After Surgery and Fast Track Surgery Pathways on Healthcare-associated Infections: Results From a Systematic Review and Meta-analysis. Ann Surg. 2017; 265: 68-79.

22. Lagoutte N, Facy O, Ravoire A, Chalumeau C, Jonval L, Rat P, Ortega-Deballon P. C-reactive protein and procalcitonin for the early detection of anastomotic leakage after elective colorectal surgery: pilot study in 100 patients. J Visc Surg. 2012; 149: e345-9.

23. Fleischmann E, Lenhardt R, Kurz A, Herbst F, Fulesdi B, Greif R. Nitrous oxide and risk of surgical wound infection: a randomised trial. Lancet 2005; 366: 1101-7.

24. Hanley JA, McNeil BJ. The meaning and use of the area under a receiver operating characteristic (ROC) curve. Radiology 1982; 143: 29-36.

25. Wacker C, Prkno A, Brunkhorst FM, Schlattmann P. Procalcitonin as a diagnostic marker for sepsis: a systematic review and meta-analysis. Lancet Infect Dis 2013; 13: 426-35.

26. Mimoz O, Benoist JF, Edouard AR, Bohuon C, Samii K. Procalcitonin and C-reactive protein during the early posttraumatic systemic inflammatory response syndrome. Intensive Care Med 1998; 24: $185-8$.

27. Aikawa N, Fujishima S, Endo S, Sekine I, Kogawa K, Yamamoto Y. Multicenter prospective study of procalcitonin as an indicator of sepsis. J Infect Chemother 2005: 11; 152-9.

28. den Dulk M, Noter SL, Hendriks ER, Brouwers MA, van der Vlies CH, Oostenbroek RJ. Improved diagnosis and treatment of anastomotic leakage after colorectal surgery. Eur J Surg Oncol 2009; 35: 420-6.

29. Tsuchida T, Le K, Okuse C, Hirose M, Nishisako H, Torikai K, et al. Determining the factors affecting serum presepsin level and its diagnostic utility: A cross-sectional study. J Infect Chemother 2021; 27: 585-91.

Saito J, Hashiba E, Mikami A, Kudo T, Niwa H, Hirota K. Pilot Study of Changes in Presepsin Concentrations Compared With Changes in Procalcitonin and C-Reactive Protein Concentrations After Cardiovascular Surgery. J Cardiothorac Vasc Anesth 2017; 31: 1262-7.

\section{Tables}

Table 1 Clinical characteristics of patients with and without infectious complications 


\begin{tabular}{lccc}
\hline & Non-infected & Infected & \\
& $\mathrm{N}=87$ & $\mathrm{~N}=27$ & P Value \\
\hline Age, yr & $69.1 \pm 11.5$ & $63.7 \pm 13.2$ & 0.044 \\
Male, \% & $62(71)$ & $17(62)$ & 0.414 \\
BMI (kg/m2) & $23.2 \pm 3.7$ & $22.7 \pm 2.7$ & 0.592 \\
ASA PS I / I /II & $0 / 65 / 22$ & $2 / 20 / 5$ & 0.033 \\
Diagnosis & & & \\
$\quad$ Cancer, N (\%) & $82(94.2)$ & $26(96.3)$ & 0.677 \\
Location of the disease & & & \\
Rectum, N (\%) & $43(49.4)$ & $20(74.0)$ & 0.024 \\
Surgical intervention & & & 0.107 \\
Right hemicolectomy, N (\%) & $24(27.5)$ & $3(11.1)$ & \\
Sigmoidectomy, N (\%) & $16(18.3)$ & $4(14.8)$ & \\
Total colectomy, N (\%) & $3(3.4)$ & $0(0.0)$ & \\
Anterior resection, N (\%) & $23(26.4)$ & $6(22.2)$ & \\
APR, N (\%) & $14(16.0)$ & $8(29.6)$ & \\
ISR, N (\%) & $6(6.8)$ & $6(22.2)$ & \\
Surgical approach & & & \\
Laparoscopy, N (\%) & $61(70.1)$ & $11(40.7)$ & \\
Length of hospital stay (d) & $12.6 \pm 7.1$ & $24.5 \pm 29.6$ & \\
\hline
\end{tabular}

Data: mean \pm SD, BMI: body mass index,

ASA PS: American Society of Anesthesiologists Physical Status

ISR: intersphincteric resection, APR: abdominoperineal resection.

Table 2 ROC curve predictions of presepsin, CRP and procalcitonin (PCT) values based on the presence or absence of infectious complications 


\begin{tabular}{|c|c|c|c|c|c|c|c|}
\hline & & Preoperative & POD1 & POD2 & POD3 & POD4 & POD6 \\
\hline \multirow{5}{*}{ Presepsin } & Cut-off $(\mathrm{pg} / \mathrm{ml})$ & 197 & 269 & 308 & 184 & 394 & 299 \\
\hline & AUC & 0.65 & 0.63 & 0.69 & 0.67 & 0.73 & 0.82 \\
\hline & $95 \% \mathrm{Cl}$ & $0.54-0.44$ & $0.50-0.75$ & $0.57-0.81$ & $0.55-0.79$ & $0.62-0.85$ & $0.73-0.91$ \\
\hline & Sensitivity (\%) & 44.4 & 69.2 & 66.7 & 85.2 & 55.6 & 73.1 \\
\hline & Specificity (\%) & 79.3 & 59.8 & 70.9 & 42.4 & 87.2 & 81.0 \\
\hline \multirow{5}{*}{ CRP } & Cut-off (mg/dl) & 0.815 & 8.425 & 17.105 & 14.08 & 10.29 & 7.394 \\
\hline & AUC & 0.58 & 0.7 & 0.75 & 0.81 & 0.86 & 0.91 \\
\hline & $95 \% \mathrm{Cl}$ & $0.46-0.7$ & $0.57-0.83$ & $0.66-0.85$ & $0.72-0.90$ & $0.78-0.94$ & $0.83-0.98$ \\
\hline & Sensitivity (\%) & 29.6 & 63.0 & 74.1 & 81.5 & 77.8 & 81.5 \\
\hline & Specificity (\%) & 89.7 & 76.7 & 66.7 & 71.8 & 81.6 & 88.4 \\
\hline \multirow{5}{*}{ РCT } & Cut-off (ng/ml) & 0.03 & 3.81 & 0.29 & 0.21 & 0.15 & 0.09 \\
\hline & AUC & 0.51 & 0.59 & 0.6 & 0.63 & 0.67 & 0.75 \\
\hline & $95 \% \mathrm{Cl}$ & $0.38-0.64$ & $0.46-0.72$ & $0.48-0.73$ & $0.51-0.75$ & $0.56-0.78$ & $0.65-0.86$ \\
\hline & Sensitivity (\%) & 44.4 & 30.8 & 70.4 & 74.1 & 77.8 & 92.3 \\
\hline & Specificity (\%) & 69.0 & 89.7 & 50.6 & 51.7 & 52.9 & 43.5 \\
\hline
\end{tabular}

\section{Figures}

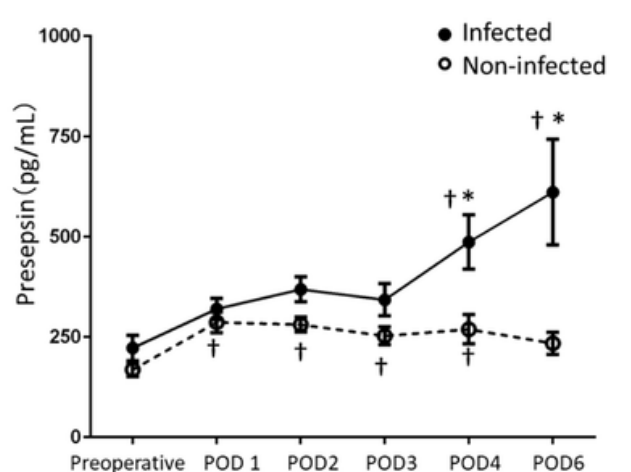

Infected: $\mathbf{p}<0.0001$

Time: $p<0.0001$

Interaction: $\mathrm{p}<0.0001$

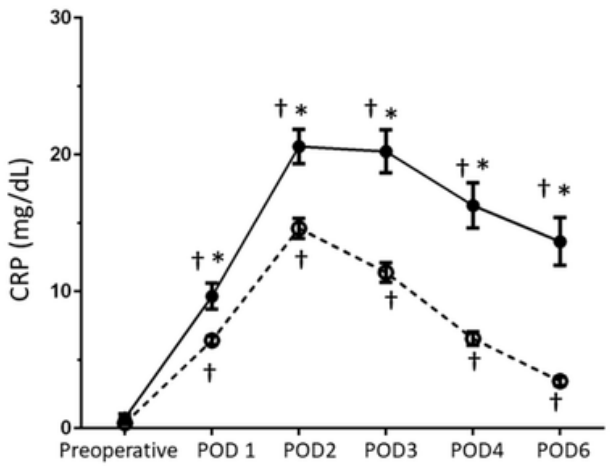

Infected: $\mathrm{p}<0.0001$

Time: $p<0.0001$

Interaction: $p<0.0001$

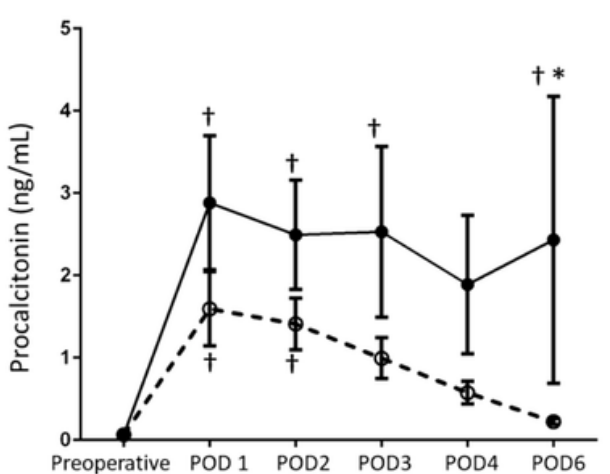

Infected: $p=0.0139$

Time: $p<0.0001$ Interaction: $\mathrm{p}<0.0001$

\section{Figure 1}

Time course of plasma concentrations of presepsin, CRP and procalcitonin Plasma concentrations of presepsin, CRP and procalcitonin before surgery and on postoperative day 1, 2, 3, 4, and 6. Data are shown as median and SEM. Two-way analysis of variance (ANOVA) with repeated measures was done on log-transformed data. There were significant differences between groups for presepsin, CRP, and procalcitonin concentrations. ${ }^{*} p<0.05$ between groups $+p<0.05$ versus preoperative 